\title{
Profile of Dermatophytic infections in G overnment Tiruvannamalai medical college, Thiruvannamalai
}

\author{
Sripriya C S*, K avitha K, Vikram balaji R, Pandian J, \\ Shanmugam K, Sivakumar D.
}

Associate Professor, Department of Microbiology, Government Thiruvannamalai Medical

College, Thiruvannamalai.

\begin{abstract}
Dermatophyte infections are extremely frequent worldwide and their epidemiological features vary according to the geographical area. Background: This study was undertaken to determine the prevalence of dermatophytosis and the spectrum of ringworm fungi in patients attending a tertiary hospital. M aterials \& M ethods: Samples were collected from 200 patients. A portion of each sample was examined microscopically and the remaining portion of each sample was cultured onto plates of Sabouraud's dextrose agar containing chloramphenicol with and without cycloheximide. Dermatophyte isolates were identified by studying macroscopic and microscopic characteristics of their colonies. Results: Out of 200 samples, fungi were detected in $148(74 \%)$ by $\mathrm{KOH}$ while $158(79 \%)$ samples were culture positive of which 130 isolates were dermatophytes. Among dermatophyte isolates T. rubrum was the most common (36\%) cause of infection. Tinea unguium was the predominant clinical manifestation accounting for $38 \%$ of the cases. T. rubrum was the most common pathogen in Tinea unguium and Tinea capitis, whereas $T$. mentagrophytes was the most common pathogen in tinea pedis. Conclusion: Further intensive epidemiological studies of dermatophytosis which have public health significance are needed.
\end{abstract}

K eywords: Dermatophytes, T. rubrum, T. mentagrophytes, Tinea unguium, T. pedis

\section{Introduction}

Dermatophytosis is an age old documented disease with references in ancient literature too. Ringworm, also known as dermatophytosis or tinea, is a fungal infection of the skin. The name "ringworm" is a misnomer, since the infection is caused by a fungus, not a worm. Ringworm infection can affect both humans and animals. The infection initially presents with red patches on

*Author for Correspondence. E-mail: sripriyamicro@gmail.com

www. ijamicro.com 
affected areas of the skin and later spreads to other parts of the body. The infection may affect the skin of the scalp, feet, groin, beard, or other areas.

Superficial mycoses are among the most frequent forms of human infections, affecting more than $20-25 \%$ of the world's population [1]. They are predominantly caused by a group of closely related keratinophilic mycelia fungi (dermatophytes) in the genera of Trichophyton, Microsporum, and Epidermophyton. These groups of fungi invade the stratum corneum of the skin or other keratinized tissues derived from the epidermis such as hair and nails [2, 3]. The Distribution, frequency, etiological agents of Dermatophytosis vary according to the geographical region studied, the climatic variations, the socioeconomic level of the population, the time of study, the presence of Domestic animals and age of the individual [9]. According to Havlickova et al. [1] the prevalence of dermatophytosis has significantly reduced in many developed nations of the world compared to the developing ones due to improved social, economic, health care, and hygiene practice factors, evident in the former. This study was conducted in Thiruvannamalai, primarily an agriculturist population rich area, where socioeconomic constraints and other common prevalent health issues have led to a low awareness of dermatophytosis by physicians and general population. Hence conducting further studies to know the actual magnitude of dermatophytosis as well as the spectrum of its etiological agents among the general population is of the highest priority.

\section{Materials and M ethods}

A total of 200 clinical samples were collected from patients visiting the Dermatology Department of Government Thiruvannamalai medical college, Thiruvannamalai. The samples were collected from January 2018 to September 2018. Before collecting the sample the infected area was cleaned with $70 \%(\mathrm{v} / \mathrm{v})$ ethanol. Then skin and finger nail samples were collected by scrapping of lesion with sterile blade and dull broken hairs from the margin of scalp lesion with forceps and transferred to sterile folded papers. Each of these papers was appropriately labeled with the age, sex, date of collection, code of a patient, and location of infection and taken to the Microbiology Laboratory immediately.

A portion of each sample was mounted in a drop of an aqueous solution of $10 \%(\mathrm{w} / \mathrm{v})$ potassium hydroxide $(\mathrm{KOH})$ on a clean microscopic slide. After 5 minutes of mounting, the preparation was examined under low $(\times 10)$ and high $(\times 40)$ power magnification for the presence of fungal elements. The remaining portion of each clinical sample was cultured irrespective of the negative or positive direct microscopic examination results onto plates of Sabouraud's dextrose agar containing chloramphenicol with and without cycloheximide and incubated for 4-6 weeks at 25$30 \circ \mathrm{C}$ aerobically. Culture plates were examined twice a week for any fungal growth. Colonies suspected of Dermatophytes were sub cultured into potato dextrose agar (Oxoid, Basingstoke, England) by slide culture technique for the production of spores and the colonies were identified by examining macroscopically (Texture, rate of growth, topography, and pigmentation of the front and the reverse side of the culture were employed for the macroscopic identification.) and microscopic characteristics by Lactophenol Cotton Blue Stain. 


\section{Results:}

In the present study a total of 200 clinical samples were collected from suspected cases of dermatophytosis of which $87(43.5 \%)$ were from males and $113(56.5 \%)$ from females.

\section{Table 1: Demographic characteristics of respondents presented for Diagnosis}

\begin{tabular}{|c|c|c|}
\hline AGE GROUPS (YEARS) & MALE & FEM ALE \\
\hline $0-10$ & 7 & 8 \\
\hline $11-20$ & 9 & 11 \\
\hline $21-30$ & 10 & 17 \\
\hline $31-40$ & 18 & 16 \\
\hline $41-50$ & 26 & 31 \\
\hline $51-60$ & 8 & 11 \\
\hline $61-70$ & 6 & 8 \\
\hline $71-80$ & 3 & 6 \\
\hline $81-90$ & 0 & 5 \\
\hline
\end{tabular}

Fungal elements were detected in 148 (74\%) of clinical samples by $\mathrm{KOH}$ wet mount while 158 (79\%) clinical samples were culture positive of which 130 isolates were dermatophytes.

Fig 1: Total no of Samples vs. Total no of positive isolates in $\mathrm{KOH} \& \mathrm{C}$ ulture.

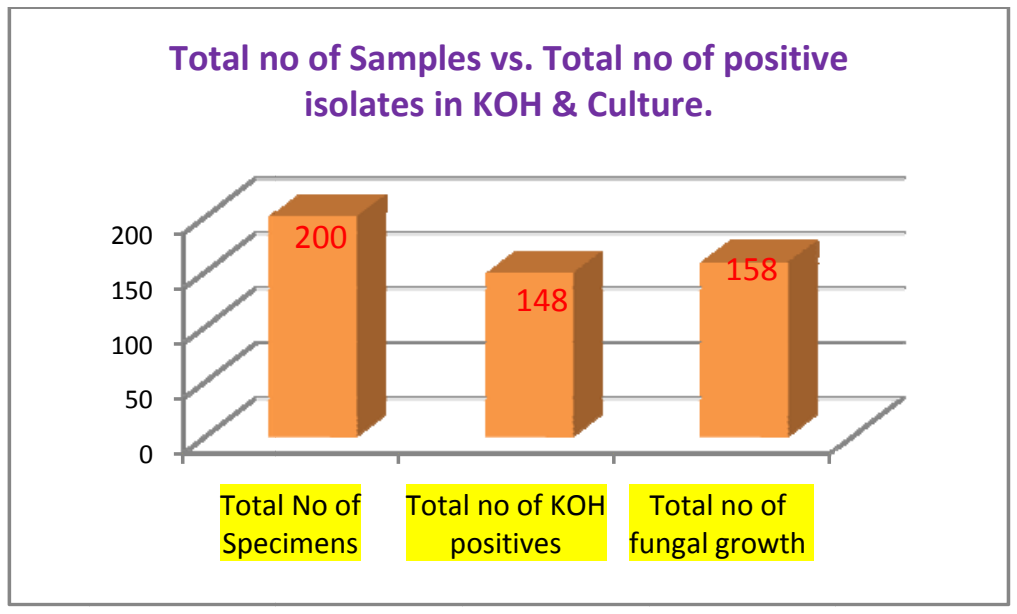


Tinea unguium was the predominant clinical manifestation accounting for $38.5 \%$ of the cases. This was followed by tinea capitis and tinea corporis accounting for $35 \%$ and $16 \%$ of the cases, respectively.

Table 3: Clinical variants of Dermatophytosis

\begin{tabular}{|l|c|c|}
\hline Clinical types & Nos. & Percentage \\
\hline T.capitis & 45 & 34.61 \\
\hline T.corporis & 21 & 16.16 \\
\hline T.cruris & 6 & 4.61 \\
\hline T.unguiium & 50 & 38.47 \\
\hline T.pedis & 8 & 6.15 \\
\hline
\end{tabular}

Further identification of dermatophytic fungi showed the presence of Trichophyton mentagrophytes (Fig 1.) Trichophyton rubrum,(Fig 2.) Trichophyton tonsurans, Trichophyton violaceum, Epidermophyton floccosum (Fig 3.) Microsporum canis (Fig 4.) and Microsporum gypseum. Among all the dermatophyte isolates T. rubrum, was the most common (36\%) cause of infection, followed by T. mentagrophytes $(22 \%)$ and T. tonsurans $(18 \%)$, whereas $M$. canis $(2 \%)$ was the least common. According to species frequency in different areas of involvement, $T$. rubrum was the most common pathogen in tinea unguium and tinea capitis, whereas $T$. mentagrophytes was the most common pathogen in tinea pedis.

Table 4: I solation Frequency of Dermatophytes

\begin{tabular}{|l|c|c|}
\hline Fungal Isolates & No of I solates & Percentage \\
\hline T.rubrum & 47 & 36 \\
\hline T.mentagrophytes & 28 & 22 \\
\hline T.tonsurans & 23 & 18 \\
\hline T.violaceum & 21 & 16 \\
\hline E.floccossum & 3 & 2 \\
\hline M.gypseum & 5 & 4 \\
\hline M.canis & 3 & 2 \\
\hline
\end{tabular}




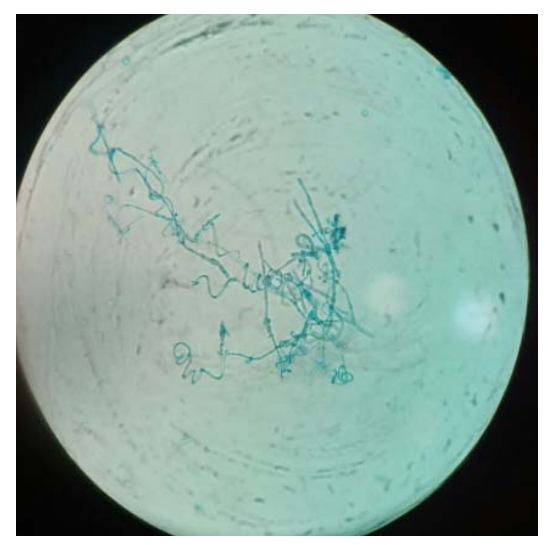

Fig 1. Trichophyton mentagrophytes

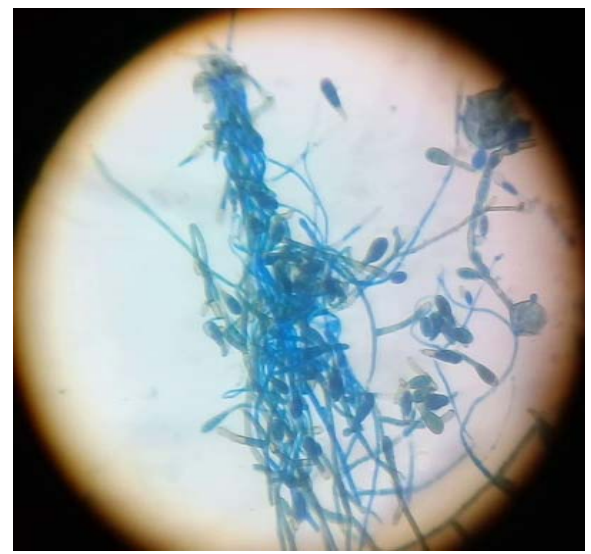

Fig 3. Epidermophyton floccosum

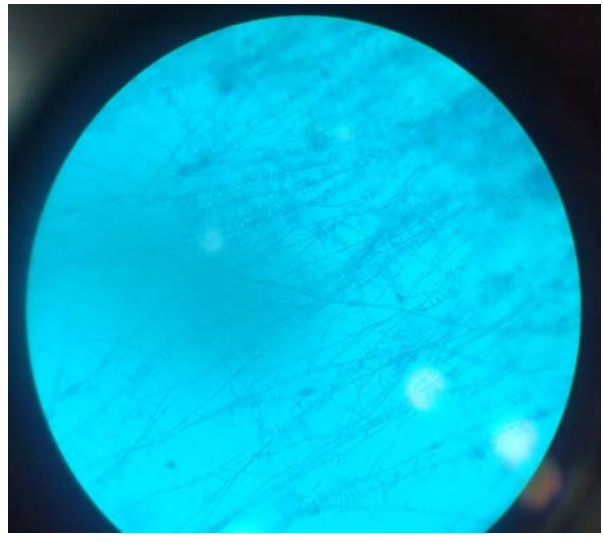

Fig 2. Trichophyton rubrum

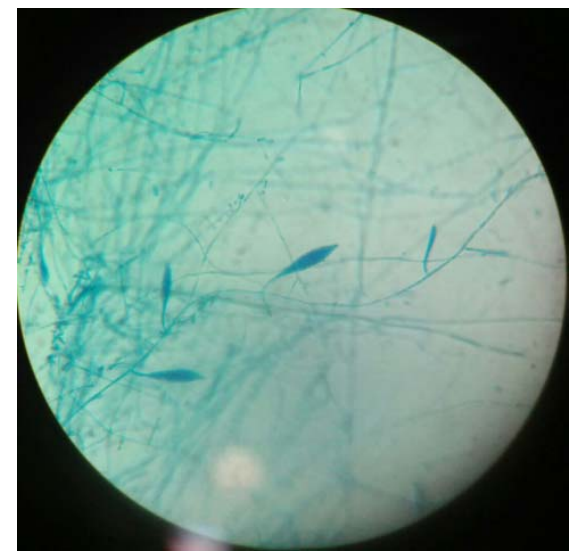

Fig 4. Microsporum canis

\section{Discussion}

Dermatophytic infections are more prevalent in the developing world and the infection is increasing in this part of the world. The present study attempted to determine the dermatophyte infections in patients attending a tertiary teaching hospital in Thiruvannamalai. Of the 200 clinical samples collected from patients with cases of suspected dermatophytosis referred to the Department of Dermatology, in the period of January 2018 to September 2018, Fungal elements were detected in 148 (74\%) of clinical samples by KOH wet mount while 158 (79\%) clinical samples were culture positive of which 130 isolates were dermatophytes A prevalence rate of $\mathrm{KOH}$ proven dermatophyte infections ranging from $53.1 \%$ to $100 \%$ and a prevalence rate of culture proven dermatophytic infections ranging from $52.2 \%$ to $67.1 \%$ have been reported by Kannan et al. [3] and by Ellabib and Khalifa [4]. Disparity in the prevalence rates of 
dermatophytosis in different studies could result from differences in the lifestyle, socioeconomic conditions, risk factors associated with study subjects, and environmental factors of study area $[1$, 2].

The present study showed that more females were affected by dermatophytes than males, with female-male ratio being 1.3:1. Earlier studies also indicated a higher prevalence of dermatophytes in females compared to males [5-8]. Meanwhile some other earlier studies recorded a higher prevalence of dermatophytes in males than females [9,10]. The predominant clinical manifestations of dermatophytosis vary considerably in different studies reported in literature. In a study conducted in India, tinea corporis $(35.4 \%)$ was the predominant clinical condition followed by tinea cruris (16.8\%) and tinea capitis (16.7\%) [5].

Similar study conducted in Iran between March 2005 and March 2007 by Rassai et al. [6] revealed that tinea cruris and tinea corporis were the most common clinical manifestation. A 7year (1997-2003) survey of dermatophytoses in Crete, Greece, conducted by Maraki et al. [7] revealed that tinea unguium was the predominant clinical manifestation. A study carried out by Devliotou- Panagiotidou et al. [8] between 1981 and 1990 in Greece depicted that tinea pedis was the most frequent clinical manifestation. Adefemi et al. [9] reported tinea capitis as a predominant clinical manifestation.

In our study, Tinea unguium was the dominant clinical manifestation involving $38.5 \%$ of the total cases of dermatophytosis, similar to many other reports $[15,18]$. Tinea capitis was the second clinical manifestation accounting for $34.5 \%$ of dermatophytosis as has been observed in other studies $[9,11,12]$. Tinea corporis was the third common clinical presentation accounting for $21 \%$ and this clinical manifestation has been reported as a dominant clinical manifestation by earlier similar studies .As universally reported by most of the workers, tinea capitis is an infection of childhood. In the present study a total of 45 patients with tinea capitis, of which 21 patients were in age group of 1-14. Similar results were reported by earlier researches $[13,14]$.The changing pattern of hormones after puberty [15] and production of inadequate amounts of inhibitory fatty acids before puberty [16] are responsible for a decrease of tinea capitis with age. On the other hand, tinea unguium was more frequent in the elderly population with an age group of 25-64. Reduced growth rate of the ungual plate, an increase in trauma rates, poor peripheral circulation, and inability to maintain good foot care could be attributed to this [17].On the other hand tinea pedis was a dominant clinical manifestation in age group 45-64 years which was in agreement with the findings of Lange et al. and Caputo et al. [18,1].

Of the total number of 130 dermatophytes isolates in the present study $71 \%$ was accounted by $T$. rubrum, T. mentagrophytes, and T. tonsurans. Among the three dominant species, T. rubrum accounted for $32 \%$ of the total isolates and our finding was compatible to studies conducted in several Asian countries $[17,20,21]$. the heterogeneity in the distribution of dermatophytosis, their etiologic agents, and the predominating clinical manifestation patterns in different parts of the world have been attributed to factors of geographic location, climate, overcrowding, health care, immigration, environmental hygiene culture, and socioeconomic conditions . 


\section{Conclusion}

This study has revealed that the prevalence of microscopic and culture confirmed dermatophytic infections in the study subjects was high. The present study has also depicted that tinea unguium was the dominant clinical manifestation involving $38 \%$ of the total cases of dermatophytosis. Of the total number of 130 dermatophyte isolates $76 \%$ was accounted for by T. rubrum, $T$. mentagrophytes, and T. tonsurans. Among the three dominant species, T. rubrum accounted for $36 \%$ of the total isolates. Because of the psychological effects and high morbidity in terms of loss of working days and treatment dermatophytic infection is a public health problem. Therefore, to obtain a true representation of the overall disease pattern of the country more such types of studies should be conducted.

\section{Conflict of Interest: NIL}

\section{References:}

1. Havlickova, B., Czaika, V. A., Friedrich, M. 2008. Epidemiological trends in skin mycoses worldwide. Mycoses, 51, 2-15.

2. Ameen, M. 2010. Epidemiology of superficial fungal infections. Clinics in dermatology, 28(2), 197-201.

3. Kannan, P., Janaki, C., Selvi, G. S. 2006. Prevalence of dermatophytes and other fungal agents isolated from clinical samples. Indian Journal of Medical Microbiology, 24(3), 212.

4. Ellabib, M. S., Khalifa, Z., Kavanagh, K. 2002. Dermatophytes and other fungi associated with skin mycoses in Tripoli, Libya. Mycoses, 45(3-4), 101-104.

5. Balakumar, S., Rajan, S., Thirunalasundari, T., Jeeva, S. 2012. Epidemiology of dermatophytosis in and around Tiruchirapalli, Tamilnadu, India. Asian Pacific Journal of Tropical Disease, 2(4), 286-289.

6. Rassai, S., Feily, A., Derakhshanmehr, F., Sina, N. 2011. Some epidemiological aspects of dermatophyte infections in Southwest Iran. Acta Dermato venerologica Croatica, 19(1), 13-15.

7. Maraki, S., Nioti, E., Mantadakis, E., Tselentis, Y. 2007. A 7-year survey of dermatophytoses in Crete, Greece. Mycoses, 50(6), 481-484.

8. Devliotou-Panagiotidou, D., Koussidou-Eremondi, T., Badillet, G. 1995. Dermatophytosis in northern Greece during the decade 1981-1990: Dermatophytosen in Nordgriechenland während der Dekade 1981-1990. Mycoses, 38(3-4), 151-157.

9. Adefemi, S. A., Odeigah, L. O., Alabi, K. M. 2011. Prevalence of dermatophytosis among primary school children in Oke-oyi community of Kwara state. Nigerian Journal of Clinical Practice, 14(1).

10. Vena, G. A., Chieco, P., Posa, F., Garofalo, A., Bosco, A., Cassano, N. 2012. Epidemiology of dermatophytoses: retrospective analysis from 2005 to 2010 and comparison with previous data from 1975. Microbiologica-Quarterly Journal of Microbiological Sciences, 35(2), 207. 
11. Al-Fouzan, A. S., Nanda, A., Kubec, K. 1993. Dermatophytosis of children in Kuwait: a prospective survey. International journal of dermatology, 32(11), 798-801.

12. Chepchirchir, A., Bii, C., Ndinya-Achola, J. O. 2009. Dermatophyte infections in primary school children in Kibera slums of Nairobi. East African medical journal, 86(2).

13. Attapattu, M. C. 1989. A study of tinea capitis in Sri Lanka. Journal of Medical and Veterinary Mycology, 27(1), 27-32.

14. Al Sheikh, H. 2009. Epidemiology of dermatophytes in the eastern province of Saudi Arabia. Research Journal of microbiology, 4(6), 229-34.

15. Oliveira, J. A. A. D., Barros, J. D. A., Cortez, A. C. A., Oliveira, J. S. R. L. D. 2006. Superficial mycoses in the City of Manaus/AM between March and November/2003. Anais Brasileiros de Dermatologia, 81(3), 238-243.

16. David, G., Richard, C. B., John, F., Michael, R. B. Medical Microbiology: A Guide to Microbial Infections: Pathogenesis, Immunity, Laboratory Diagnosis and Control, Churchill Livingstone, 16th edition, 2003.

17. Kaur, R., Kashyap, B., Bhalla, P. 2008. Onychomycosis-epidemiology, diagnosis and management. Indian Journal of Medical Microbiology, 26(2), 108.

18. Lange, M., Nowicki, R., Barańska-Rybak, W., Bykowska, B. 2004. Dermatophytosis in children and adolescents in Gdansk, Poland. Mycoses, 47(7), 326-329.

19. Caputo, R., De Boulle, K., Del Rosso, J., Nowicki, R. 2001. Prevalence of superficial fungal infections among sports-active individuals: results from the Achilles survey, a review of the literature. Journal of the European Academy of Dermatology and Venereology, 15(4), 312-316.

20. Ali-Shtayeh, M. S., Salameh, A. A., Abu-Ghdeib, S. I., Jamous, R. M., Khraim, H. 2002. Prevalence of tinea capitis as well as of asymptomatic carriers in school children in Nablus area (Palestine) Häufigkeit von Tinea capitis und asymptomatischen Trägern bei Schulkindern in der Nablus-Region (Palastina). mycoses, 45(5-6), 188-194.

21. Hussain, I., Aman, S., Haroon, T. S., Jahangir, M., \& Nagi, A. H. 1994. Tinea capitis in Lahore, Pakistan. International journal of dermatology, 33(4), 255-257. 